\title{
REFRACTIVE SURGERY IN CHILDREN
}

Ophthalmology Clinics of North America - Volume 14, Issue 3 (September 2001) - Copyright (C) 2001 W. B. Saunders Company - About This Journal

DOI: $10.1016 /$ S0896-1549\%2805\%2970244-3

\section{ARTICLES}

\section{REFRACTIVE SURGERY IN CHILDREN}

\section{Arlene V. Drack MD 1 Paolo Nucci MD 2}

1. Department of Ophthalmology, Emory University School of Medicine, Atlanta, Georgia

${ }_{2}$ University of Milan, Milan, Italy

This work was funded in part by the Georgia Lions Children's Eyecare Center

Address reprint requests to

Arlene V. Drack, MD

Associate Professor Dept. of Ophthalmology

Emory University School of Medicine

4 th Floor Building B

1365 Clifton Ave

Atlanta, GA 30322

Twenty years from now, the use of refractive surgery to treat pediatric conditions will have been shown to be either a great advance, a sometimes useful tool, or a serious mistake for young patients with refractive errors. It falls on ophthalmologists practicing in the early part of the 21 st century to determine how to best serve, as well as protect patients while navigating through those years. The aim of this chapter is to analyze refractive surgery techniques in light of current understanding of the anatomy and physiology of the growing pediatric eye, to learn from the lessons already reported for the various techniques, and to discuss both the potential benefits and possible pitfalls of using refractive surgery for a variety of indications in pediatric ophthalmology.

\section{NATURAL HISTORY OF REFRACTION}


At birth, the normal human infant corneal power averages 51 diopters; lens power averages 34 diopters. The corneal diameter averages 10 $\mathrm{mm}$, and the axial length $17 \mathrm{~mm}$. This very short eye with a rather round lens and small, steep cornea, rapidly changes shape and size over the first two years of life. By age 2, the corneal diameter has expanded to $12 \mathrm{~mm}$ and has flattened to the adult range of 42 to 44 diopters. The axial length is $95 \%$ of its adult size of $24 \mathrm{~mm}$. The lens flattens to 28 diopters by 6 months of age, and continues to grow in size throughout childhood. ${ }_{[10]}$ The endothelial cell layer of the cornea has its highest cell density during intrauterine life. Since endothelial cells cease to divide very early in development, the rapid expansion of the cornea and anterior chamber precipitates a dramatic and continuous drop in endothelial cell density over the first few years of life, as the existing cells move and thin to cover a larger area. [22] The rigidity of the sclera and cornea change markedly from birth to adulthood, becoming progressively less malleable and deformable with increasing age. The vitreous changes consistency from a firm gel in infancy and childhood, to a partial liquid in adulthood.

Normal human infant eyes average between 1.25 and 2 diopters of hyperopia at birth; the shorter axial length is not completely neutralized by the steeper cornea and rounder lens. Emmetropization occurs as the three main components of refraction-corneal curvature, axial length, and lens power-grow and change, ideally balancing each other in power to arrive at plano refraction. Almost certainly these physical traits, and the way they interact, are inherited via the interplay of several different genes, possibly modified by environmental factors. Plano refraction usually is reached by 9 to 12 years of age, with little change after the age of 13 years. In those destined to be hyperopic, greater than normal amounts of hyperopia are present at birth, and the usual rate of decline in hyperopia is not of sufficient magnitude to result in plano by the time the eye has finished its growth. Since children have very large accommodative amplitudes (approximately 15 diopters at age 5 years, compared to 6 diopters at age 40), children who are moderately hyperopic usually see normally. In some cases, however, a high accommodative convergence to accommodative amplitude (AC/A ratio) may render even a young hyperopic child symptomatic because of induced esotropia.

Since accommodation is a central process, the amount of accommodation must be almost equal between the two eyes. Most people can differentially accommodate 0.50 diopters between their eyes. Therefore, anisohyperopia of an amount significantly greater than this can be symptomatic, since the visual system automatically accommodates the least amount that will produce a clear image. The less hyperopic eye is thus preferentially stimulated, whereas the more hyperopic eye is blurred. This is an important cause of amblyopia.

Astigmatism, a difference in refraction between the two major axes of the cornea, is often present at birth, but in many cases resolves. Abnormalities of the orbits or eyelids may induce astigmatism, but most cases are idiopathic and sometimes inherited. Significant amounts of astigmatism that are present after 1 year of age are usually permanent. However the visual significance is related to the magnitude of the cylinder, whether it is symmetric between the two eyes, and the age of the child. Children and young adults can often comfortably use accommodation to place the conoid of Sturm on the macula and achieve good vision without correction for small-to-moderate amounts of astigmatism, as long as it is similar in both eyes.

Myopia can result from excessive axial length, excessive corneal steepness, an unusually round lens, or simply a mismatch between the various components in a given eye. Simple myopia usually develops between 9 and 12 years of age because of progressive axial elongation. Children destined to be myopic usually do not have the normal amount of hyperopia during infancy and early childhood.

Refractive errors can occur because of abnormal size or shape of the ocular components at birth, a lack of correlation of the components of refraction, a failure of one or more of the components to grow in the usual way, or a failure of the components to grow and change in complementary ways (failure of emmetropization). The majority of refractive errors seen throughout the human life span are predestined at birth and develop as the eye rapidly grows and changes throughout the first 13 years of life.

\section{HISTORY OF REFRACTIVE CORRECTION}

Refractive errors have probably existed as long as human beings have, but it is difficult to discern when spectacles were first invented. There is good evidence that about 1287 AD, spectacles were invented in Northern Italy, but they may have been already in use in China and other parts of Asia. [25] Early spectacles were made with frames of leather or bone, sometimes suspended from hats or hair or held in place by hand. Lenses were made of quartz or beryl. Most early lenses were for presbyopia. For many years only plus and minus lenses were known, thus, while myopia and hyperopia could be corrected, luckless astigmats were forced to rely on the conoid of Sturm. Eventually cylindrical lenses were developed.

The invention of bifocals by Benjamin Franklin in the mid 1700s brought convenience to presbyopes, ${ }_{[25]}$ and the development of lightweight, high-index plastics continued to improve spectacle lens design.

The next revolution occurred when contact lenses were invented in 1888. The first contact lenses were made of glass and covered the sclera 
as well as cornea. In the 1930s, contact lenses were developed in plastic and began to gain popularity. With the change from scleral to corneal lenses in the 1940s, reduced corneal edema and increased wearing time led to the widespread use of contact lenses as a primary treatment for refractive errors. $[25]$

Contact lenses continued as the mainstay of refractive error correction until the 1970s, when radial keratotomy began to gain popularity. Surgeons had experimented with incisions to alter the corneal curvature to reduce refractive errors since the 19 th century. Sato began using posterior corneal incisions in Japan in 1939, but on long-term follow-up, most eyes developed corneal edema. [29] Yenaliev modified the incisions, making them anterior, and did surgery on many patients in Russia from 1969 through 1977. The technique was further modified in Russia by Fyodorov and Durnev who did animal, then human studies during the 1970s. In 1978 Bores started to perform radial keratotomy in the United States. ${ }_{[29]}$ The technique involves making radial, partial thickness cuts in the peripheral cornea. These cuts flatten the central cornea, thereby reducing or eliminating myopia. The cuts also destabilize the cornea, however, and refractive shifts over time are common, as is corneal edema at high altitudes. ${ }_{[17]}[18][30]$

A companion technique that makes the cornea steeper in order to treat hyperopia, called hexagonal keratotomy, proved disastrous in many eyes, as did some other techniques for treating hyperopia. ${ }_{[31]}$ Epikeratophakia, a procedure in which a donor lenticule specific refractive power is sewn onto the cornea, was popularized during the 1980s, especially for pediatric aphakia. [20] [28] Despite encouraging results in some patients, interface problems between the graft and the host cornea, and large myopic shifts over time, have caused the procedure to be largely abandoned in favor of intraocular lenses or contact lenses. Modifying the corneal shape with hard contact lenses and with heat (thermal keratoplasty) have also been tried with little long term success.

Laser refractive surgery revolutionized the field in the 1990s. Photorefractive keratectomy (PRK), a procedure in which the corneal epithelium is removed, and a laser utilized to ablate corneal stroma thereby causing flattening, was found to produce fairly stable correction of mild-to-moderate myopia. The procedure is painful, however, and has a long recovery period, with many patients experiencing transient or sometimes permanent corneal haze. Laser in situ keratomieusis (LASIK), a technique in which a microkeratome is used to raise a partial thickness corneal flap, and the exposed stroma is laser ablated and the flap replaced, was the next development. It has proven to be very popular for the treatment of mild to moderately high myopia and low hyperopia. Because the epithelium remains intact, there is little pain post-operatively. Stability appears to be good, and although there can be complications, such as displaced or lost flap, interface debris or inflammation, and regression over time, it has been deemed safe enough and to provide a rapid enough recovery to perform the procedure on both eyes of a patient on the same day, if desired. Other procedures for higher degrees of myopia, such as use of intraocular anterior chamber or posterior chamber phakic lenses, have been associated with higher complication rates, including cataract formation. [27]

\section{REFRACTIVE SURGERY IN CHILDREN}

Many studies have been done in adults to evaluate the safety, efficacy, and complications, as well as indications for treatment using refractive surgery techniques. As the procedures have become more widely used and accepted, the lower age limit for treatment has been questioned by practitioners and patients. Laser refractive surgery is approved by the Food and Drug Administration (FDA) for use in only in patients 18 years of age or older. For some lasers and some indications, the lower age limit is 21 years. Clinically, however, the age at which patients are being considered for refractive surgery is dropping, but without the concomitant studies that were performed in adults. As detailed earlier, given the vast differences between the growing and changing pediatric eye and visual system, and the fully formed counterpart in the adult, there is little reason to believe that safety and efficacy studies in the latter are relevant in the former. Indeed, our experience with pediatric keratoplasty, cataract surgery, and intraocular lens implantation has been that different techniques and postoperative care are necessary in pediatric eyes. Even the prescribing of contact lenses and glasses, including the indications, what is prescribed and when, are very different for children than adults. The constant battle against amblyopia, the child's hyperactive immune system, and the unknowns of how an intervention will affect normal growth all must be considered.

Many drugs and devices are never tested in children, and therefore never approved for use in pediatrics by the FDA. Pediatric practitioners have long been compelled to use such modalities "off label" to enable their young patients to benefit from innovations. But great caution must exercised in these situations.

Epikeratophakia was one of the first modern refractive surgeries to be performed in children. Used largely for infants and children with aphakia, especially if contact lens intolerant, results were mixed. ${ }_{[20]}^{[28]}$ The lenticule-cornea interface often became hazy, making retinoscopy difficult and compromising vision. Amblyopia sometimes developed on the basis of poor media clarity rather than uncorrected aphakia, substituting one problem for another. Large myopic shifts developed over time, causing anisometropia again. Most importantly, 
the main selling point, namely that removing the contact lens as a variable would improve compliance with patching, did not prove to be true. One study showed that the visual outcome in children with unilateral cataract was more related to patching compliance, rather than whether the aphakia was treated with a contact lens versus epikeratophakia. ${ }_{[8]}$

The next attempt at refractive surgery in children was again aimed at aphakia. Intraocular lens implantation as a primary treatment after cataract surgery in children older than 2 years of age, and usually as a secondary procedure in children who had been rendered aphakic in infancy, was adapted slowly and carefully to pediatric cases. The IOLAB study ${ }_{[34]}$ showed good outcomes without insurmountable complications for children older than 1 year of age, as did many smaller series. However, many of the complications and issues, such as dealing with the posterior capsule, choice of lens power, and postoperative care, proved to be unique to children. Further information on the evolution of IOL's in children can be obtained in the Fallaha chapter.

PRK and LASIK are now being offered to some children under the age of 18. No multi-center, controlled studies have been done to evaluate the safety, efficacy, or long-term outcomes. There are, however, a number of small series reviewed here.

Several studies have reported improvement in vision in the amblyopic eye of children treated with laser refractive surgery for

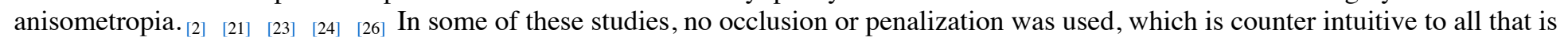
known about amblyopia in daily practice and reported in the literature. In all of these studies, adult nomograms for the amount of ablation required to correct various refractive errors were accurate in children. Average correction achieved was also similar to that found in adult studies. Complications included deposits under the flap in one child who had LASIK ( 1 of 14 children in the study), ${ }_{[24]}$ and corneal haze in $10 \%,{ }_{[26]} 16 \%,[2]$ and $40 \%$ [21] of those who had PRK. There was no long-term follow up, and no data were reported on endothelial cell counts, future axial length growth, or changes in corneal curvature over more than one or two years. $\operatorname{Rashad}_{[24]}$ treated 14 eyes with unilateral high myopia and amblyopia with LASIK. Topical steroids were used for five weeks postoperatively. Part-time occlusion was continued for all children 9 years of age or younger. All procedures were done using inhalation Sevoflurane anesthesia. There was regression of the effect up to at least three months postoperatively. Keratometry results showed regression until six months postoperatively. Patients were followed for one year. At the end of this time, the average central corneal thickness was reduced from $498.1+/-23.9 \mu \mathrm{m}$ to $431.3+/$-28.9. Preoperatively, uncorrected visual acuity was 20/400 or worse in all eyes, with best corrected ranging from 20/40 to 20/100. At one year post surgery, uncorrected vision ranged from 20/20 to 20/70, with all eyes gaining at least two lines. All eyes had best corrected vision of 20/40 or better post-operatively, including children who continued occlusion after surgery. The authors credit the improved vision to less minification, fewer optical aberrations, and full-time correction of the refractive error. In a study by Nucci and Drack, 14 eyes of 14 patients aged 9 to 14 years were treated with PRK or LASIK in one eye for unilateral high myopia. ${ }_{\text {[23] }}$ All patients had already attempted and terminated occlusion therapy. Although the amount of refraction correction achieved was excellent, and there were no untoward complications, only two amblyopic eyes improved their best corrected vision by two lines post-operatively. These were the two best seeing eyes pre-operatively, and they improved from 20/80 to 20/60. Average best corrected vision before surgery was 20/147; after surgery, it was 20/121. Whether this represents true reversal of amblyopia or simply optical improvement cannot be determined. No patient achieved better stereopsis, and no one developed new or resolved a pre-existing strabismus. All procedures were performed using topical anesthesia (Fig. 1) . In this study refractive surgery was shown to be safe and effective (though PRK patients required topical steroids for three months, and LASIK patients had a more marked myopic shift postoperatively than PRK patients, despite similar preoperative refractive stability) for reducing myopia. It also demonstrated that using refractive surgery to correct anisometropia at an age when children are cooperative and can have the procedure performed under topical anesthesia is unlikely to treat amblyopia.

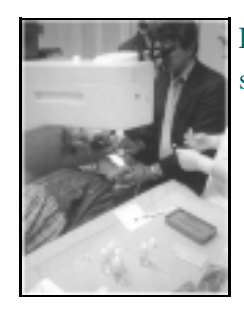

Figure 1. An 8-year-old child cooperates during a photorefractive keratectomy procedure using topical anesthesia. Patient coaching, careful patient selection, and parental encouragement make general anesthesia unnecessary in most cases.

\section{INDICATIONS FOR REFRACTIVE SURGERY IN CHILDREN}

In a poll of 34 ophthalmologists attending a workshop on pediatric refractive surgery during the 2000 annual meeting of the American Association for Pediatric Ophthalmology and Strabismus, approximately two thirds of the respondents said that anisometropia would be their number one indication for considering refractive surgery in a child. Only one respondent felt that there were no valid indications for 
refractive surgery in children. Other possible indications cited included hyperopia associated with accommodative esotropia, bilateral refractive amblyopia intolerant of spectacles or contact lenses, stable myopia, and special activity requirements for good uncorrected vision. $[6 \mathrm{~A}]$

The main reason for pediatric ophthalmologists' interest in additional treatments for anisometropia is, of course, amblyopia. Visual development is a dynamic process in infants and children. The brain requires a clear image to build appropriate neural connections with the eyes, and if one eye sends a clearer picture, the brain preferentially chooses that signal, resulting in poor vision in the other eye. If neither eye sends a clear picture, poor vision will develop in both eyes. This type of vision loss is called amblyopia, and it is reversible only until approximately 9 years of age. In animal models in which one eye is sutured closed shortly after birth to create deprivation amblyopia similar to congenital cataracts, it has been demonstrated that the ocular dominance columns in the visual cortex corresponding to the deprived eye shrink, while those for the normal eye enlarge. ${ }_{[13]}$ However, most amblyopia is caused by asymmetric refractive errors or strabismus, not deprivation. Staining of the striate cortex was done post-mortem in one adult with longstanding anisometropic amblyopia, and no such changes were found, suggesting that anisometropia may cause amblyopia by a different mechanism than deprivation. [11] A similar study done on the striate cortex of a woman who had developed dense amblyopia secondary to accommodative esotropia also

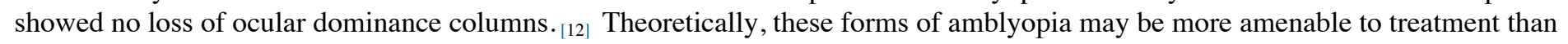
deprivation amblyopia, which drastically alters the architecture of the visual cortex. This may explain why refractive correction alone often reverses amblyopia in young children with this condition.

Bilateral high myopia of greater than 6 diopters, bilateral hyperopia of greater than 5 diopters, and astigmatism greater than 2 diopters bilaterally can cause bilateral amblyopia.

Anisometropia is a major risk factor for amblyopia. However, the age at which it is detected, the degree, and the type of refractive error(s) present all play a role in how great the risk is. Many children who are anisometropic at 1 year of age will equalize their refractions by 10 years of age. However, $90 \%$ of children with 3 diopters or more spherical equivalent anisometropia at the age of 1 year will not, and $60 \%$ of these will develop amblyopia. [1]

In young children, refractive amblyopia can often be treated by optical correction alone. Children who present at older ages, or with very large anisometropias, usually require patching in addition to optical correction for resolution. Overall, the success rate for 20/40 vision or better utilizing optical correction with or without patching has been reported as $66.7 \%$ in a meta-analysis of published studies. [9] The success rate was $83 \%$ in a large study at the University of Iowa. ${ }_{[14]}$

Anisoastigmatism of more than one diopter can cause amblyopia, but unilateral high astigmatism is uncommon, and would be an uncommon indication for refractive surgery in children.

Anisohyperopia causes amblyopia, because accommodation is a binocular phenomenon. As discussed above, most people can compensate for approximately 0.50 diopters of anisohyperopia. Larger amounts of anisohyperopia are, therefore, potentially amblyogenic. In one large series, the average difference between the eyes in anisohyperopic amblyopes was +2.46 diopters. ${ }_{[14]}$ Another study found that $0 \%$ of children with anisohyperopia of 0 to 0.5 diopters developed amblyopia; $8 \%$ of those with 0.5 to 1 diopter developed it, and this steadily rose to $100 \%$ of those with more than 3 diopters of anisohyperopia. ${ }_{\text {[32] }}$ The larger the anisohyperopia, the greater the relative blur and the denser the amblyopia. Often, greater amounts of anisometropia also correlate with poorer compliance with spectacle wear, especially if the less hyperopic eye is close to plano, since children perceive that they see well without correction, having adapted to monocular vision. Since this is a problem usually detected in early childhood, contact lens wear is often impractical and unsuccessful. One potential use of refractive surgery in children would be treating the more hyperopic eye of such patients to equalize refractive errors. The benefit would be removing spectacle wear compliance from the equation, leaving parents free to concentrate solely on penalization or occlusion of the other eye. The child would also appear more normal, unlike the situation in which one eye is magnified by a high plus lens. The risks of doing this procedure immediately upon diagnosis, when the benefits would be greatest, are theoretically high. Performing refractive surgery before the age of 6 or 7 years requires general anesthesia in most children. This carries additional morbidity and mortality risks far beyond those of spectacle wear, or even refractive surgery at an older age when topical anesthesia is possible. There is no way of predicting what effect reshaping the cornea will have on an actively growing eye. Most eyes emmetropize during this time. Would treated eyes develop a myopic shift? Would refractive surgery for that myopia be possible in the future? Some of the most serious complications encountered with refractive surgery, occasionally requiring penetrating keratoplasty, have occurred with attempted correction of hyperopia. And many of the well-known complications, such as epithelial ingrowth, occur more frequently after repeat procedures. Thus, recommending a surgical procedure with a high likelihood of a requisite repeat, and also a higher likelihood of complications with subsequent surgeries, must be carefully considered. Although some children present compliance problems using spectacles to treat anisohyperopia, most do not. Amblyopia is safely reversed, and as children enter adolescence, a contact lens can be used rather than spectacles. 
A more conservative indication in the same population would be anisohyperopia in children greater than 9 years of age who have successfully completed amblyopia therapy and wish to discard their anisometropic spectacles. In a study of 46 eyes with primary hyperopia or hyperopic astigmatism treated with LASIK, $63 \%$ had a refraction +/- 1 diopter at six months postoperatively. The pre-operative range of hyperopia was +0.50 diopters to +6.00 diopters. One eye lost two lines of best corrected visual acuity.[16] Another requirement could be sufficient cooperation to do the procedure under topical anesthesia. Typically in adult refractive surgery, stability of refraction is a requirement. This would be unobtainable in many hyperopic children, and would either exclude most of them, or force a new type of criteria to be developed. Before discarding this requirement, one should ask why it has been put in place for adults. If it is sound for adult eyes, it probably should be followed for pediatric cases as well, disqualifying most pediatric hyperopes, whether amblyopic, anisometropic or otherwise. The unknown long-term effects on the growth of the eye, the unknown ultimate refractive outcomes, the known risks, and the alternative of contact lens wear until adulthood with possible refractive surgery when eye growth is completed should all be discussed. Ideally, a prospective, controlled study should be done with a well-informed group before the procedure is offered as a treatment.

Anisomyopia presents a different picture. When this condition is significant enough to cause amblyopia, one eye is usually highly myopic from birth, the other often close to emmetropia. Unlike typical juvenile onset myopia, unilateral high myopia is rarely progressive during childhood. And unlike anisohyperopia, compliance is very frequently poor. Unilateral high myopia has the worst prognosis for good vision of any refractive amblyopia. . 14$]_{3}$ This may be partly because unilateral high myopia necessitates one high minus spectacle lens which minifies images, as well as the relatively high incidence of associated organic retinal and optic nerve anomalies. Weakley ${ }_{[32]}$ found that among 138 patients with anisomyopia, none with less than 2 diopters difference developed amblyopia, but all of those above this amount did. However, in another large series from Iowa, the average amount of anisomyopia causing amblyopia was -8.05 diopters. ${ }_{[14]}$ One indication for refractive surgery in children could be unilateral high myopia with amblyopia, intolerant of contact lens or spectacle wear. Techniques for correcting myopia are better tested in adults than those for hyperopia, and eyes amblyopic from myopia are almost uniformly legally blind when compliance is poor, thus the risk of losing vision is very small. Since these eyes do not tend to change refraction over time, the need for repeat procedures is not preordained as in childhood hyperopia. There are still several controversial issues, however. Although small studies have shown that correction can be achieved in older children, there are no long-term studies of stability. In addition, to treat the amblyopia, the procedure would have to be performed in infancy or early childhood, necessitating general anesthesia, with all of the caveats mentioned previously. Ideally, before recommending this procedure with its attendant risks even in this condition where we often have so little to offer, a prospective study should be performed to insure that the results have reasonable stability and no unforeseen consequences in children.

Another possible indication for refractive surgery is accommodative esotropia. Again, a potential pitfall in using this technique in children is that the natural history of the hyperopia causing the esotropia is to resolve or lessen on its own. Intervening with refractive surgery before the age of 13 years might destine children to develop high myopia in later years as their axial lengths followed their previous growth curves. Refractive surgery after the age of 13 years is less likely to result in later myopia, but may cause unstable strabismus if the hyperopia is of too high a degree to completely correct. As mentioned previously, hyperopia has had a history of more complications associated with refractive surgery procedures, especially if the hyperopia is high. For this reason high hyperopes might end up under-corrected. This may convert a tropia to a phoria, or may not resolve the esotropia at all. A young, partially corrected hyperope with accommodative esotropia may appear straighter even if under-corrected, but may lose stereopsis, and even vision, if amblyopia develops from a microtropia monofixator status. These concerns must be addressed by practitioners familiar with pediatric ophthalmology issues. A recent letter to a refractive surgery journal noted that "because even moderate amounts of hyperopia are well tolerated by children, the target refractive endpoint when treating hyperopes need not ... be emmetropia." ${ }_{[6]}$ Patients with high AC/A ratios would be poorly served if this were assumed to be true in all cases. If anisometropia is induced, even to a relatively small degree, it may precipitate switching fixation, making dissociated deviations that were previously latent, manifest. For those patients older than 13 years with stable refractions, good stereopsis and low amounts of hyperopia, refractive surgery could be considered. A prospective study following changes in alignment and stereopsis, as well as subsequent ocular growth and health would be helpful to both patients and practitioners.

The most controversial indication for refractive surgery is amblyopia in older children who have failed patching, as well as contact lens or spectacle treatment. Clinical practice and basic research data support the existence of a critical period of visual development during which amblyopia can develop and be reversed, and after which neither is possible. It is also clear from clinical practice as well as the literature that significant amblyopia usually does not resolve without patching or penalization. Yet the current literature is rife with amblyopia "cures" from refractive surgery in older children. First, it should be noted that there are some children in whom amblyopia treatment is effective well past the usually quoted age of 9 years, and anisometropic amblyopia is the most amenable to late treatment. [19] [33] Even a small percentage of adults demonstrate improved vision in their amblyopic eye, if the better eye is lost. ${ }_{[7]}$ Experiments have already been discussed in which it has been shown that the ocular dominance columns do not contract in anisometropic amblyopia as they do in the deprivation type, possibly explaining why many children with this type of amblyopia recover with spectacle correction alone. However, most reports of amblyopia cures with refractive surgery in older children are improvements of about two lines of best corrected vision. This can be attributable to better optics, and even better effort after the experience of surgery. If the common definition of amblyopia as a 
difference in vision between the two eyes of two or more lines is used, these eyes still have amblyopia. Whether improvements in vision in some of these eyes are due to partial reversal cannot be known, and should not be claimed, unless vision becomes essentially equal. Alternatively, occlusion or penalization could be used after refractive surgery to demonstrate whether more improvement in amblyopia could be obtained.

The group of children most likely to be interested in refractive surgery are those with simple myopia. No lower age limit has been uniformly agreed upon for performing refractive surgery in these patients. Since their main goal in wanting the surgery is to enjoy clear vision without spectacles or contact lenses, the requirement commonly used for adults that the refraction must be stable for at least a year seems reasonable. If strictly adhered to, it will eliminate most myopic youngsters from consideration, since the average change per year through adolescence is -0.50 diopters per year. Many myopes continue progressing well into their $20 \mathrm{~s}$ and even $30 \mathrm{~s}$. Performing refractive surgery at early ages therefore, should be accompanied by informed consent, noting that contact lenses or spectacles are likely to be needed again in the future, or more surgery with its attendant increased risks will be needed. In addition, it is unknown whether having refractive surgery on an eye with increasing myopia would increase or in other ways alter the usual myopic shift.

\section{IF REFRACTIVE SURGERY, WHICH REFRACTIVE SURGERY?}

Currently, the main options for refractive surgery are intracorneal rings, PRK, LASIK, phakic intraocular lenses, and clear lens extraction. Intracorneal rings have the proported advantage of being reversible, but it is unknown whether the original corneal shape will be resumed, especially in growing eyes. In addition, a foreign body in the anterior part of the eye of an active youngster may be more prone to breakage, expulsion, or predisposition to infection. Finally, the rings best correct lower amounts of myopia, so they have limited usefulness in treating the amblyogenic variety.

PRK is more painful immediately post-operatively, but with the use of nonsteroidal anti-inflammatories, the pain is easily controlled in children and appears to be less intense than in adults. (personal experience of PN). A bandage contact lens can be used for the first three to five days. One potential problem is the long course of topical steroids necessary to avoid haze, often up to three months. This necessitates frequent intraocular pressure checks to be sure steroid responsive glaucoma is not developing. Detecting this glaucoma may be especially difficult given recent reports of falsely low readings using conventional tonometry methods after refractive surgery, leading to missed

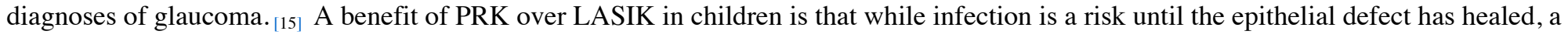
displaced or lost flap in LASIK is a far more devastating and likely problem, given children's propensity to rub their eyes.

LASIK requires more cooperation on the part of the patient and has greater risks because of the possibility of displaced flaps. However, it has the benefits of being able to correct fairly high refractive errors with a quick recovery and the ability to operate on both eyes if necessary at one sitting, avoiding the amblyopia risk entailed in doing one eye at a time to maintain some vision and avoiding two painful eyes with PRK.

With either refractive laser procedure there are potential problems with doing the procedure under general anesthesia in that awake patients are able to fixate during the procedure, ensuring that the correct optical center is chosen. Decentration has been reported to be a problem even in awake children, and has been reported to cause diplopia due to prismatic effects when it has been induced in adults. ${ }_{[3]}$

Clear lens extraction carries an increased risk of retinal detachment in highly myopic eyes. ${ }_{[5]}$ Given the exuberant inflammatory response that follows lens extraction in children, the high (approximately 30\%) ${ }_{[4]}$ risk of glaucoma following lens extraction in children, the risk of subsequent hyperopia, and the loss of accommodation, this option should probably be considered very rarely in children.

Phakic intraocular lenses have a significant risk of secondary cataracts. ${ }_{[27]}$ The pediatric anterior chamber is smaller than the adult, making crowding and possible corneal and angle compromise more likely. Given the complexities of cataract surgery and post-operative care in children, the known as well as theoretical risks of this procedure should be weighed in children.

\section{SUMMARY}

Refractive surgery techniques, especially those using laser ablation, have revolutionized the treatment of refractive errors. The short-term results have been well studied in adults, but long-term outcomes are not known. No good studies exist to tell us whether the pediatric eye 
responds the same as the adult eye to these techniques, but there is reason to believe that the pediatric eye will have many differences, both short- and long-term based on other similar surgeries adapted for pediatrics. These techniques have great potential to add to our armamentarium of treatments for frustrating problems such as unilateral high myopia with amblyopia, but they should be used with caution. The patients who are the most attractive to refractive surgeons, namely, teenagers with typical myopia who want to discard their spectacles, are the patients with potentially the most to lose from a procedure with unknown long-term results for a condition easily treated with other modalities. Those who need it most, namely infants and children with high anisometropia who cannot tolerate contact lenses or spectacles, are the least cooperative, the most difficult to treat postoperatively, and the least able to afford expensive treatments not covered by insurance, are therefore the least likely to be offered the procedure, or to have a study designed to evaluate their specific needs and concerns. It behooves ophthalmologists interested in pediatrics to carefully discuss and research the possible indications and theoretical concerns of these powerful techniques in pediatric eyes.

\section{References}

1. Abrahamsson M, Sjostrand J: Natural history of infantile anisometropia. Brit J Ophthalmol 80:860-863, 1996

2. Alio JL, Artola A, Claramonte P et al.: Photorefractive keratectomy for pediatric myopic anisometropia. J Cataract Refract Surg 24:327-330, 1998 Abstract

3. Balakrishnan V, Lim AS, Tseng PS, et al: Decentered ablation zones resulting from photorefractive keratectomy with an erodible mask. Int Ophthalmol 17:179184, 1993 Abstract

4. Chrousos GA, Parks MM, O'Neill JF: Incidence of chronic glaucoma, retinal detachment and secondary membrane surgery in Pediatric Aphakic Patients. Ophthalmology 91:1238-1241, 1984 Abstract

5. Colin J, Robinet A, Cochener B: Retinal detachment after clear lens extraction for high myopia: Seven year follow up. Ophthalmol 106:2281-2284, 1999

6. Davidorf JM: Pediatric refractive surgery. J Cataract Refract Surg 26:1567-1568, 2000 Citation

6A. Drack, unpublished data

7. El Mallah MK, Chakravarthy U, Hart PM: Amblyopia: Is visual loss permanent? Br J Ophthalmol 84:944-945, 2000

8. Elsas FJ: Visual acuity in monocular pediatric aphakia: Does epikeratophakia facilitate occlusion therapy in children intolerant of contact lens or spectacle wear? J Ped Ophthalmol Strab 27:304-309, 1990

9. Flynn JT, Schiffman J, Feuer W, et al: The therapy of amblyopia: An analysis of the results of amblyopia therapy utilizing the pooled data of published studies. Trans Am Ophthamol Soc 96:431-450, 1998

10. Gordon RA, Donzis PB: Refractive development of the human eye. Arch Ophthalmol 103:785-789, 1986

11. Horton JC, Stryker MP: Amblyopia induced by anisometropia without shrinkage of ocular dominance columns in human striate cortex. Proc Natl Acad Sci U S A 90:5494-5498, 1993 Abstract

12. Horton JC, Hocking DR: Pattern of ocular dominance columns in human striate cortex in strabismic amblyopia. Vis Neurosci 13:787-795, 1996 Abstract

13. Hubel DH, Wiesel TN, LeVay S: Plasticity of ocular dominance columns in monkey striate cortex. Philosophical Transactions of the Royal Society of LondonSeries B: Biological Sciences. 278:377-409, 1977

14. Kutschke PJ, Scott WE, Keech RV: Anisometropic amblyopia. Ophthalmology 98:258-263, 1991 Abstract

15. Levy Y, Zadok D, Glovinsky Y, et al: Tono-Pen versus Goldmann tonometry after excimer laser photorefractive keratectomy. J Cataract Refract Surg 25:486491, 1999 Abstract

16. Lindstrom RL, Hardten DR, Houtman DM, et al: Six month results of hyperopic and astigmatic LASIK in eyes with primary and secondary hyperopia. Trans Am Ophthalmol Soc 97:241-255, 1999 Abstract 
17. Mader TH, White LJ: Refractive changes at extreme altitude after radial keratotomy. Am J Ophthalmol 119:733-737, 1995 Abstract 18. McDonnell PJ: Sight-threatening complications after radial keratotomy. Arch Ophthalmol 114:211-212, 1996 Citation 19. Mintz-Hittner HA, Fernandez KM: Successful amblyopia therapy initiated after age 7 years. Arch Ophthalmol 118:1535-1541, 2000 Abstract 20. Morgan KS, McDonald MB, Hiles DA, et al: The nationwide study of epikeratophakia for aphakia in older children. Ophthalmology 95:526-532, 1988 Abstract 21. Nano HD, Muzzin S, Irigaray LF: Excimer laser photorefractive keratectomy in pediatric patients. J Cataract Refract Surg 23:736-739, 1997 Abstract 22. Nucci P, Brancato R, Mets MB, et al: Normal endothelial cell density range in childhood. Arch Ophthalmol 108:247-248, 1990 Abstract 23. Nucci P, Drack AV: Refractive surgery for unilateral high myopia in children. JAAPOS, in press 24. Rashad KM: Laser in situ keratomileusis for myopic anisometropia in children. J Refract Surg 15:429-435, 1999 Abstract 25. Rosenthal JW: Spectacles and other vision aids. San Francisco, Norman Publishing, 1996, pp 17-118 26. Singh D: Photorefractive keratectomy in pediatric patients. J Cataract Refract Surg 21:630-633, 1995 Abstract 27. Trindade F, Pereira F: Cataract formation after posterior chamber phakic intraocular lens implantation. J Cataract Refract Surg 24:1661-1663, 1998 Abstract 28. Uusitalo RJ, Uusitalo HM: Long-term follow-up of pediatric epikeratophakia. J Refract Surg 13:45-54, 1997 Abstract 29. Waring GO, Moffitt SD, Gelender H, et al: Rationale for and design of the national eye institute prospective evaluation of radial keratotomy (PERK) study. Ophthalmology 90:40-58, 1983 Abstract 30. Waring GO, Lynn MJ, McDonnell PJ: Results of the prospective evaluation of radial keratotomy (PERK) study 10 years after surgery. Arch Ophthalmol 112:1298-1308, 1994 Abstract 31. Waring GO: A cautionary tale of innovation in refractive surgery. Arch Ophthalmol 117:1069-1073, 1999 Citation 32. Weakley DR: The association between nonstrabismic anisometropia, amblyopia, and subnormal binocularity. Ophthalmology 108:163-171, 2001 Abstract 33. Wick B, Wingard M, Cotter S, et al: Anisometropic amblyopia: Is the patient ever too old to treat? Optom Vis Sci 69;866-878, 1992

34. Young TL, Bloom JN, Ruttum M, et al: The IOLAB, Inc pediatric intraocular lens study. AAPOS Research Committee. American Association for Pediatric Ophthalmology and Strabismus. Journal of the American Association of Pediatric Ophthalmology and Strabismus 3:295-302, 1999

MD Consult Resources: About I Discoverability Toolkit I Blog I Newsletter | Contact Us Terms and Conditions I Privacy Policy I Registered User Agreement

\section{Copyright (C) 2012 Elsevier Inc. All rights reserved. www.mdconsult.com}

Bookmark URL: /das/journal/view/0/N/12279619?ja=275147\&PAGE=1.html\&issn=0896-1549\&source=

Client IP Address: 129.255.1.139 DAS Host: isis 
